\title{
Effect of H-Bonding on Order Amplification in the Growth of a Supramolecular Polymer in Water
}

Citation for published version (APA):

Garzoni, M., Baker, M. B., Leenders, C. M. A., Voets, I. K., Albertazzi, L., Palmans, A. R. A., Meijer, E. W., \& Pavan, G. M. (2016). Effect of H-Bonding on Order Amplification in the Growth of a Supramolecular Polymer in Water. Journal of the American Chemical Society, 138(42), 13985-13995.

https://doi.org/10.1021/jacs.6b07530

Document status and date:

Published: 26/10/2016

DOI:

10.1021/jacs.6b07530

Document Version:

Publisher's PDF, also known as Version of record

\section{Document license:}

Taverne

\section{Please check the document version of this publication:}

- A submitted manuscript is the version of the article upon submission and before peer-review. There can be important differences between the submitted version and the official published version of record.

People interested in the research are advised to contact the author for the final version of the publication, or visit the DOI to the publisher's website.

- The final author version and the galley proof are versions of the publication after peer review.

- The final published version features the final layout of the paper including the volume, issue and page numbers.

Link to publication

\footnotetext{
General rights Owners
rights.

- You may freely distribute the URL identifying the publication in the public portal. please follow below link for the End User Agreement:

www.umlib.nl/taverne-license

Take down policy

If you believe that this document breaches copyright please contact us at:

repository@maastrichtuniversity.nl

providing details and we will investigate your claim.
}

Copyright and moral rights for the publications made accessible in the public portal are retained by the authors and/or other copyright owners and it is a condition of accessing publications that users recognise and abide by the legal requirements associated with these

- Users may download and print one copy of any publication from the public portal for the purpose of private study or research.

- You may not further distribute the material or use it for any profit-making activity or commercial gain

If the publication is distributed under the terms of Article $25 \mathrm{fa}$ of the Dutch Copyright Act, indicated by the "Taverne" license above, 


\title{
$\mathrm{J}$

\section{Effect of H-Bonding on Order Amplification in the Growth of a Supramolecular Polymer in Water}

\author{
Matteo Garzoni, ${ }^{\dagger}$ Matthew B. Baker, ${ }^{\ddagger}{ }^{\S}$ Christianus M. A. Leenders, ${ }^{\ddagger}$ Ilja K. Voets, ${ }^{\ddagger}$ Lorenzo Albertazzi, ${ }^{\prime \prime}$ \\ Anja R. A. Palmans, ${ }^{\ddagger}$ E. W. Meijer, ${ }^{\ddagger}$ and Giovanni M. Pavan* ${ }^{\dagger}$ \\ ${ }^{\dagger}$ Department of Innovative Technologies, University of Applied Sciences and Arts of Southern Switzerland, Galleria 2, Via Cantonale \\ 2c, CH-6928 Manno, Switzerland \\ ${ }^{\ddagger}$ Institute for Complex Molecular Systems, Eindhoven University of Technology, 5612 AZ Eindhoven, The Netherlands \\ ${ }^{\S}$ MERLN Institute for Technology-Inspired Regenerative Medicine, Maastricht University, P.O. Box 616, 6200 MD Maastricht, The \\ Netherlands \\ "Institute for Bioengineering of Catalonia (IBEC), 08028 Barcelona, Spain
}

\section{Supporting Information}

\begin{abstract}
While a great deal of knowledge on the roles of hydrogen bonding and hydrophobicity in proteins has resulted in the creation of rationally designed and functional peptidic structures, the roles of these forces on purely synthetic supramolecular architectures in water have proven difficult to ascertain. Focusing on a 1,3,5-benzenetricarboxamide (BTA)based supramolecular polymer, we have designed a molecular modeling strategy to dissect the energetic contributions involved in the self-assembly (electrostatic, hydrophobic, etc.) upon growth of both ordered BTA stacks and random BTA aggregates. Utilizing this set of simulations, we have unraveled the cooperative mechanism for polymer growth, where a critical size must be reached in the aggregates before

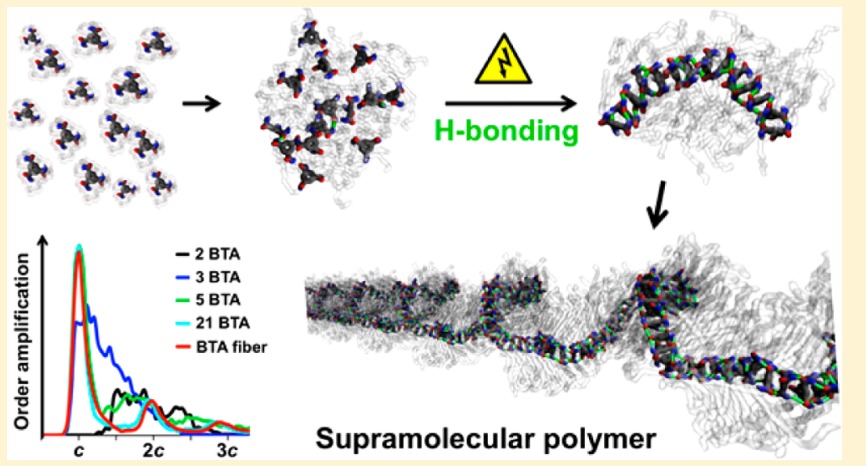
emergence and amplification of order into the experimentally observed fibers. Furthermore, we have found that the formation of ordered fibers is favored over disordered aggregates solely on the basis of electrostatic interactions. Detailed analysis of the simulation data suggests that $\mathrm{H}$-bonding is a major source of this stabilization energy. Experimental and computational comparison with a newly synthesized 1,3,5-benzenetricarboxyester (BTE) derivative, lacking the ability to form the H-bonding network, demonstrated that this BTE variant is also capable of fiber formation, albeit at a reduced persistence length. This work provides unambiguous evidence for the key $1 \mathrm{D}$ driving force of hydrogen bonding in enhancing the persistency of monomer stacking and amplifying the level of order into the growing supramolecular polymer in water. Our computational approach provides an important relationship directly linking the structure of the monomer to the structure and properties of the supramolecular polymer.
\end{abstract}

\section{INTRODUCTION}

Supramolecular polymers can mimic many of the properties of conventional covalent polymers, while allowing for dynamic, bioinspired, and adaptive properties. ${ }^{1}$ Formed via transient noncovalent interactions between monomers, dynamic behavior and reversibility are encoded into their molecular structure. These inherent properties resulted in the development of many functional materials with enhanced processing characteristics, self-healing behavior, stimuli responsiveness, and novel electronic properties. ${ }^{2}$

Supramolecular polymers are ideally suited to build new biomaterials ${ }^{3}$ that can mimic or interact with dynamic, biological environments. Attaining fundamental understanding on the interplay of different types of noncovalent interactions in relation to the dynamics of the formed materials could allow the design of functional water-soluble supramolecular poly- mers ${ }^{4}$ as dynamic as cells and the extra-cellular matrix (ECM). ${ }^{5}$ Recently, this led to the development of bioinspired supramolecular polymers based on peptide amphiphiles, ${ }^{6-8}$ ureidopyrimidinones, ${ }^{9}$ cyclodextrins, ${ }^{10,11}$ cucubiturils, ${ }^{12,13}$ dendrimers, ${ }^{14}$ and functionalized gold nanoparticles, ${ }^{15}$ to name a few.

In this framework, 1,3,5-benzenetricarboxamide (BTA)based supramolecular polymers, in which the BTA monomers self-assemble directionally due to well-defined 3-fold hydrogen bonding and stacking of cores, are an ideal scaffold for fundamental studies on supramolecular polymers. ${ }^{16}$ BTA selfassembly is well characterized in a variety of molecular architectures and environments. ${ }^{16}$ By engineering a hydro-

Received: July 27, 2016

Published: October 4, 2016 
a

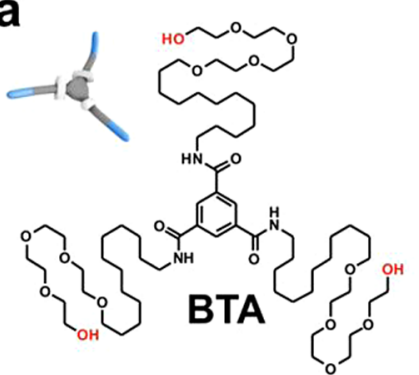

e

$\bigcirc$ BTA $_{\text {monomer }}$
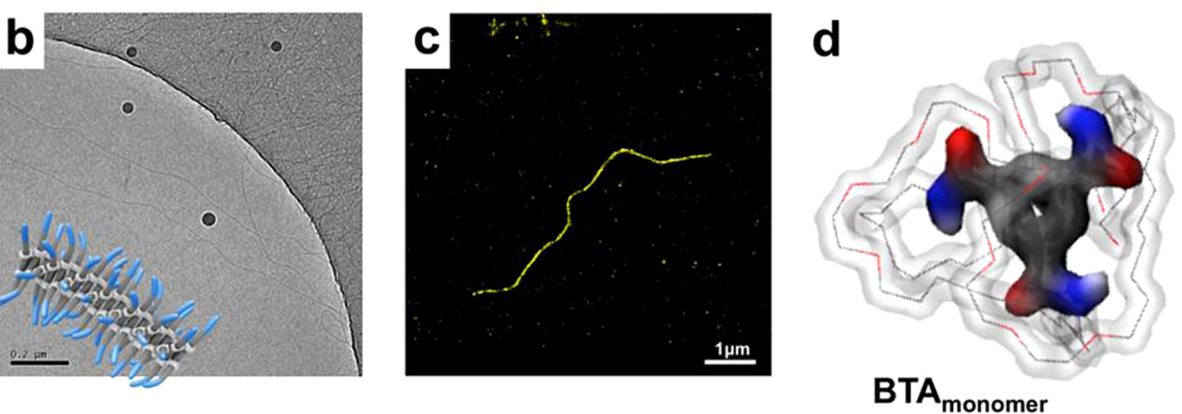

f

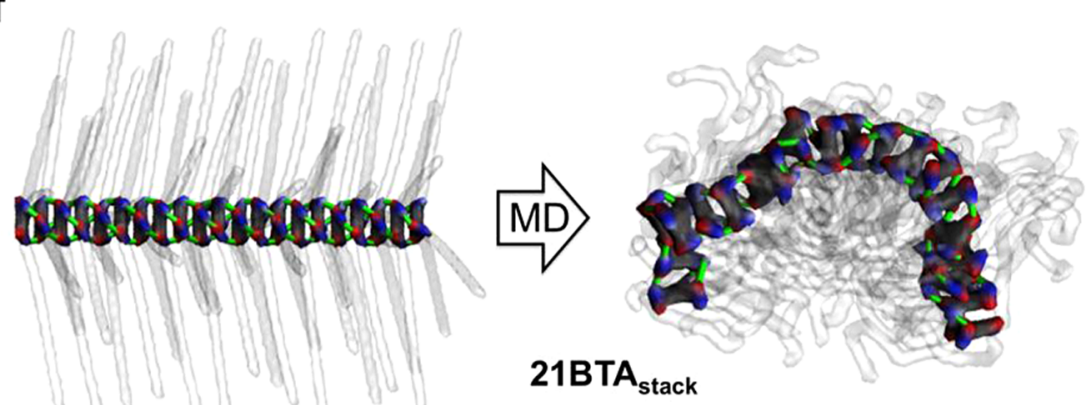

g
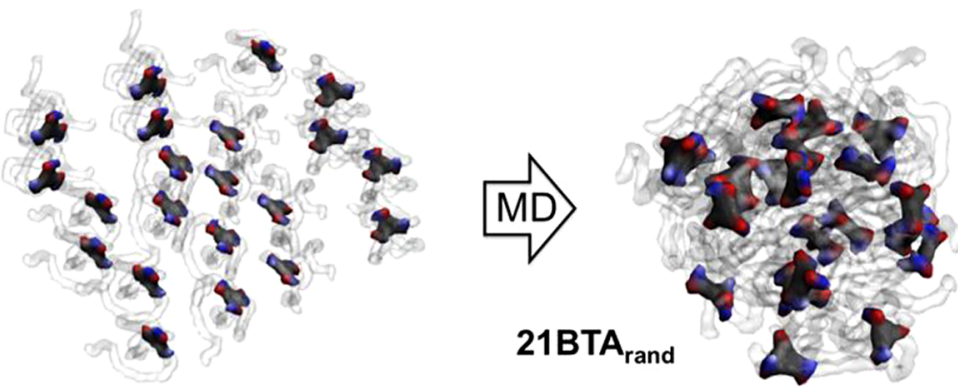

Figure 1. Modeling BTA assembly. (a) Molecular structure of the water-soluble BTA monomer studied herein. (b,c) TEM (b) and STORM (c) images of BTA fibers in water. (d) Equilibrated BTA monomer in water solution obtained from the MD simulation. (e) Modeling strategy adopted in this study. Comparison between ordered and disordered BTA assemblies of the same size (same number of BTA monomers) allows studying the modulation of the interactions leading to the growth of ordered supramolecular polymers in water. (f) Starting ( $0 \mathrm{~ns})$ and final (400 ns) configurations of the $\mathbf{2 1 B T A}_{\text {stack }}$ simulated system. $(\mathrm{g})$ Starting $(0 \mathrm{~ns})$ and final $(400 \mathrm{~ns})$ configurations of the 21BTA $\mathbf{A r n d}_{\text {ramulated system. In the }}$ snapshots, the BTA side chains are transparent, the BTA cores are colored per atom (C, gray; $\mathrm{O}$, red; and N, blue), and $\mathrm{H}$-bonds are colored in green. Water molecules are not shown for clarity.

phobic dodecyl spacer $\left(\mathrm{C}_{12}\right)$ connected to a water-compatible tetraethylene glycol tail $\left(\mathrm{EG}_{4}\right)$ around the BTA core (Figure 1a), we have recently shown that these monomers form highaspect ratio supramolecular fibers in aqueous solution (Figure $1 \mathrm{~b}, \mathrm{c}) .{ }^{17}$ This BTA analogue has been used to study the mechanism of monomer exchange, ${ }^{18}$ the ability to reorganize and cluster monomers in response to binding with multivalent recruiters, ${ }^{19}$ and how small changes in the monomer structure affect the exchange dynamics. ${ }^{20,21}$ Despite these advances, the polymerization behavior of water-soluble BTAs (and many aqueous $\mathrm{H}$-bonding-based supramolecular polymers) remains extremely difficult to interpret at a fundamental level. ${ }^{21}$ Because of the hydrophobicity of the monomers, the efficient depolymerization of such amphiphilic polymers in aqueous solution using temperature or organic solvent is prohibitively difficult. Consequently, information as to the behavior, importance, and interplay of interactions such as H-bonding and hydrophobicity becomes difficult to ascertain with conventional experimental techniques.
Of particular importance to understanding supramolecular polymers is their mechanism of growth. The two major mechanisms to date are isodesmic and cooperative polymerizations. ${ }^{22}$ In an isodesmic polymerization, the addition of each monomer to a growing polymer occurs with the same release of free-energy. On the other hand, in a cooperative polymerization, monomer addition becomes increasingly favorable with the growth of the polymer, usually after a nucleation event, while the energy gain accompanying the addition of more monomers is eventually seen to level off beyond a certain oligomer size. ${ }^{22 \mathrm{~b}}$ In general, cooperative supramolecular polymerization forms longer polymers than isodesmic polymerization if the free-energy of monomer addition to the growing chain is the same. ${ }^{22}$ Traditionally, BTA-based supramolecular polymers are cooperative in the formation of the H-bonding network in organic solvents. ${ }^{16}$ While some preliminary results suggested cooperativity in a sugar functionalized BTA in water, ${ }^{23}$ little is known about the ability of assembled BTAs to maintain their cooperative $\mathrm{H}$-bonding network in the presence of water as a competitive solvent. 
Driven by the clear challenges in obtaining insight at a molecular level into these supramolecular polymers, computer simulations have been recently introduced to complement experimental work. Previous studies based on atomistic and DFT simulations have been conducted to model stacks of smaller BTA derivatives with shorter side chains in the gas phase or in organic solvents, focusing on the study of selfassembly cooperativity in the BTA stacks. ${ }^{24-27}$ These efforts provided important insight on $\mathrm{H}$-bonding and dipole-dipole interactions in an intrinsically ordered condition, in which the short side chains and the absence of a polar solvent emphasize the level of order in the system and the role of H-bonding. However, in aqueous solution, the increased structural complexity of the water-soluble monomers (Figure 1a) and the presence of important solvophobic effects make the computational study of these supramolecular fibers drastically more complicated. ${ }^{20}$

All-atom molecular dynamics (MD) simulations recently allowed the study of self-assembled fibers of peptide amphiphiles, ${ }^{6}$ chromonic liquid crystals, ${ }^{28}$ and water-soluble BTA monomers ${ }^{20}$ in explicit water. In particular, our MD simulations permitted detailed inspection of a BTA-based supramolecular polymer in water at atomic scale and provided insight into the tertiary structure, hydrophobic versus $\mathrm{H}$ bonding interactions, and penetration of water into the structure. Our models of infinite water-soluble BTA fibers showed that these supramolecular polymers are far from being perfectly extended and ordered in water solution. Moreover, while $\mathrm{H}$-bonding remains active in the BTA polymer, the strong folding of the BTA side chains (primary folding) and of the fibers themselves (secondary folding) during the simulations demonstrates the predominant character of the hydrophobic effects. ${ }^{20}$ Comparing models of chiral and achiral monomer assemblies also allowed us to untangle the effect of a subtle mutation in the monomer on the polymer structure, which provided plausible explanations for differences in experimentally observed monomer exchange dynamics between the achiral and chiral assemblies. ${ }^{20}$

A thorough understanding of the behavior of BTA-based supramolecular polymers in water is fundamental toward the rational design of self-assembled BTA materials in biologically relevant environments. In such a complex framework, we are interested in answering fundamental questions such as: What are the factors controlling the directionality of monomers' selfassembly? What interactions control/favor the emergence of order in the polymer? What is the exact role of H-bonding in this process? While in organic solvent monomer self-assembly has been suggested to proceed in rather uniform way during polymer growth, ${ }^{16,24,25,27,29}$ is this the same in water? Does a precise limit exist (critical size) over which order emerges in the supramolecular structure, while below this threshold disordered aggregates are formed?

Herein, we report a comprehensive atomistic modeling approach to understand the factors controlling the growth of ordered supramolecular BTA polymers in water to answer these questions. Systematic all-atom MD simulations of ordered (stacked) one-dimensional (1D) BTA assemblies of incremental size, and comparison with randomly ordered BTA aggregates of the same size, allow us to obtain unique insight on the amplification of order in the BTA system. We unravel the key interactions controlling the emergence and gradual augmentation of stacking order into the BTA assemblies while their size increases, thus favoring the growth of ordered BTA polymers in water (Figure 1). Finally, experimental and computational comparison to a structurally analogous 1,3,5benzenetriester (BTE) derivative, having the amides simply replaced by esters and lacking the ability to form a strong $\mathrm{H}$ bonding network, permits us to unambiguously define the role of H-bonding in the growth of an ordered BTA supramolecular polymer in water.

\section{RESULTS AND DISCUSSION}

Cooperativity in BTA Self-Assembly in Water. The cooperative self-assembly behavior of BTAs in the gas phase or in organic media has been well studied, both experimentally ${ }^{30}$ and computationally. ${ }^{24-27,29}$ However, in water, experimental limitations prevented the unambiguous assignment of a cooperative self-assembly mechanism for the BTA described here.

Consistent with previous DFT and atomistic MD studies on BTA stacking in the gas phase $24,26,27$ and organic solvent (nonane), ${ }^{25,29}$ we built atomistic models of prestacked ordered assemblies of a water-soluble BTA (Figure 1a) in which the size of the stacks was systematically increased. In particular, we built model stacks composed of 2, 3, 5, 7, 14, and 21 initially extended BTA monomers. This series was completed by including a previously reported "infinite" fiber model composed of 48 stacked BTAs replicated along the main axis through periodic boundary conditions. ${ }^{20}$ Such infinite model has already proven useful to simulate the bulk of a BTA supramolecular polymer, $^{20}$ as well as of other supramolecular polymers in water. ${ }^{6}$ These seven prestacked assemblies $\left(\mathbf{2 B T A}_{\text {stack }}\right.$ to 48BTA $_{\text {stack }}$ Figure 1e) were equilibrated as immersed in a simulation box filled with TIP $3 \mathrm{P}^{31}$ explicit water molecules along $400 \mathrm{~ns}$ of MD simulation in NPT conditions using the AMBER 12 software. $^{32}$ During this time, all BTA assemblies rearranged and successfully reached the equilibrium in the $\mathrm{MD}$ regime (computational details are provided in the Supporting Information).

The MD simulations show that, while larger BTA stacks bend, but remain stable (e.g., Figure 1f, 21BTA stack; $_{\text {Supporting }}$ Information, 14BTA $\mathbf{A}_{\text {stack}}$ ), core stacking was found instable in the smaller aggregates. Seen in Figure 2a,b, for example, the initial stacking in the $\mathbf{2} \mathbf{B} \mathbf{T} \mathbf{A}_{\text {stack }}$ system disassembled early during the MD run. H-bonding between the two BTA monomers fails, and a single $\mathrm{H}$-bond only intermittently appears in the system (Figure $2 \mathrm{~b}$ ). In this case, the structural rearrangement and side chain folding due to hydrophobic collapse are evidently stronger than the H-bonding. Similar behavior is seen in the $\mathbf{3} \mathbf{B T A} \mathbf{A}_{\text {stack }}$ system (see the Supporting Information). Increasing the size of the stacks was found to result in increased stability of the core-core stacking. In particular, BTA stacking is found stable in aggregates with sizes $\geq$ 5BTA $_{\text {stack }}$ but only on a local basis (short-range), while globally BTA stacking was still found nonuniform and discontinuous. On the other hand, long, uniform, and persistent BTA stacks are obtained for assemblies with size $\geq$ 14BTA $_{\text {stack}}$.

Energetic and structural analyses of the $\mathrm{MD}$ simulations provide useful data to quantify these observations. From the equilibrated phase of each MD simulation (the last $100 \mathrm{~ns}$ ), we calculated the self-assembly free-energies $(\Delta G)$ of each system. All self-assembly $\Delta G$ values were calculated as the free-energy gain for $n$ monomers to stay in an assembled state rather than disassembled in solution (see methods section); the more negative/favorable was the $\Delta G$, the stronger was the assembly (values are calculated per-BTA monomer to compare between 

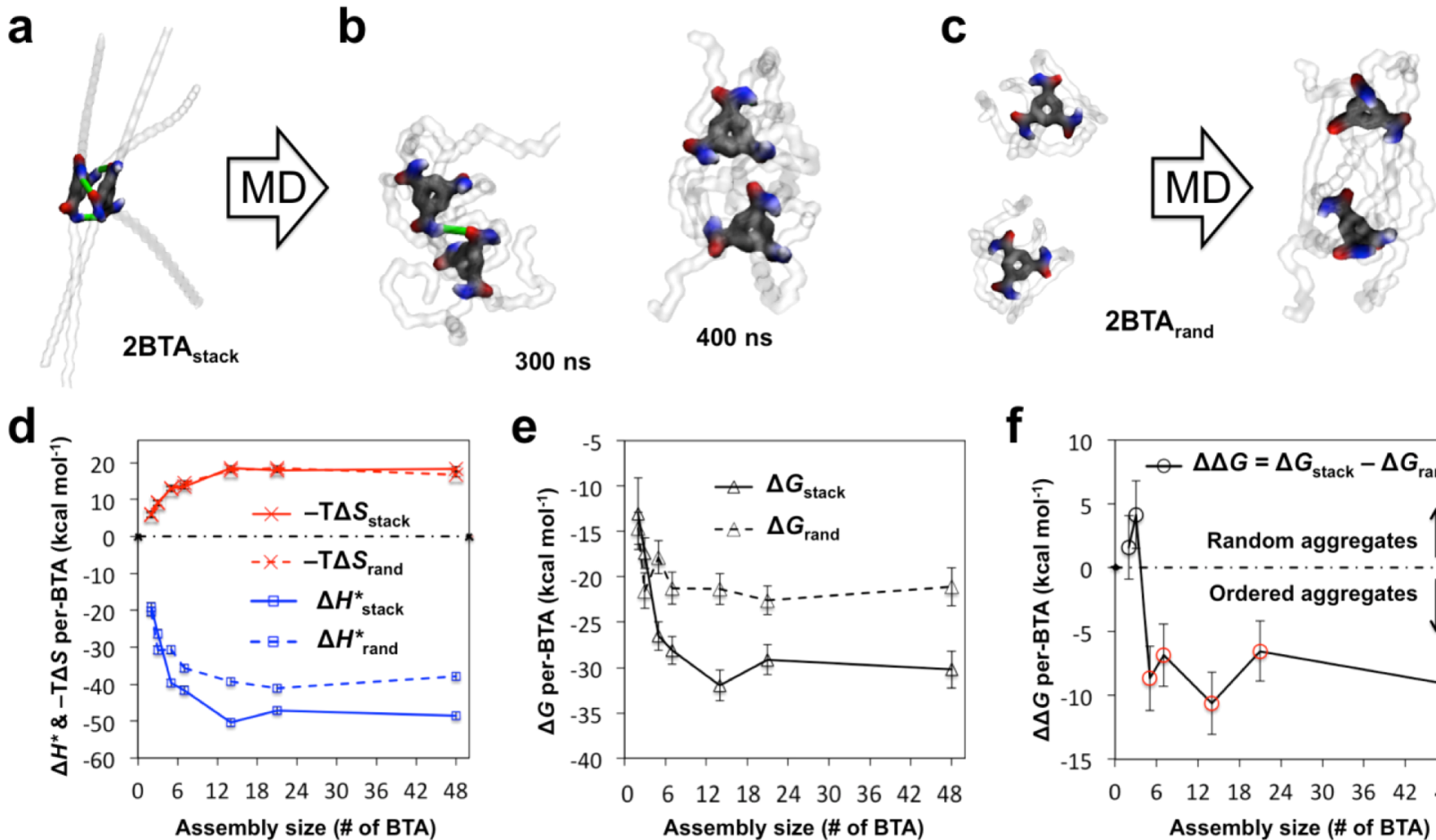

$300 \mathrm{~ns}$
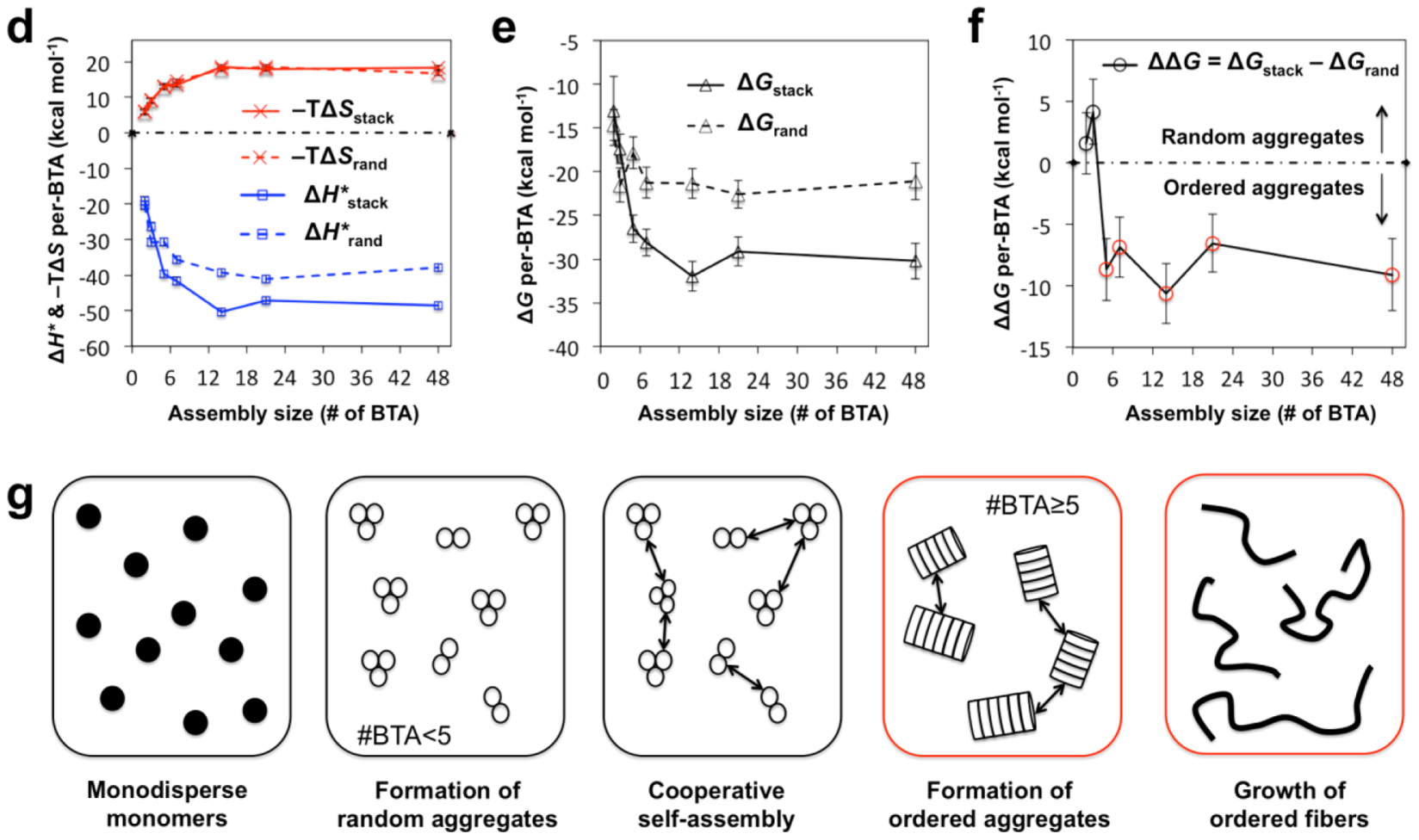

Figure 2. Self-assembly energies. ( $a, b)$ Starting (extended) and equilibrated structures taken from the MD simulation of $\mathbf{2 B T A}_{\text {stack }}$ system. During the run, the initial core stacking (a) disappears and a single H-bond (b) appears only intermittently between the BTAs. (c) Starting and final MD structure of $\mathbf{2 B T A}_{\text {rand }}$ system. (d) Per-BTA $\Delta H^{*}$ (blue) and $-T \Delta S$ data (red) for ordered $\left(\mathbf{B T A}_{\text {stack: }}\right.$ solid lines) and disordered BTA assemblies

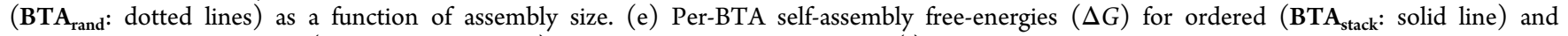
disordered BTA assemblies $\left(\right.$ BTA $_{\text {rand }}$ : dotted line) as a function of assembly size. (f) Per-BTA $\Delta \Delta G$ values. Negative $\Delta \Delta G$ values indicate that formation of ordered BTA aggregates (stacks) is energetically favored over disordered (random) ones. (g) Scheme illustrating the mechanism of formation of ordered BTA supramolecular polymers in water deduced from the MD data.

different size assemblies). The $\Delta G$ was calculated as $\Delta G=$ $\Delta H^{*}-T \Delta S$, where the $-T \Delta S$ term is related to the entropy variation of the solute due to monomer aggregation, and the $\Delta H^{*}$ term, capturing both nonbond solute-solute $\left(\Delta E_{\text {gas }}\right)$ and solute-solvent $\left(\Delta G_{\text {solv }}\right)$ interactions, is directly related (albeit not exactly equivalent) to the self-assembly enthalpy of our systems (see methods section and Supporting Information). Particularly interesting for this work, $\Delta E_{\text {gas }}$ includes the van der Waals $\left(\Delta E_{\mathrm{vdW}}\right)$ and electrostatic $\left(\Delta E_{\text {ele }}\right)$ nonbonding interactions between the BTA monomers. In general, the assemblies modeled herein are characterized by favorable $\Delta H^{*}\left(\Delta H^{*}<0\right.$ : interactions between the BTAs are augmented in the assembly compared to a disperse solution), and an unfavorable entropic term $(-T \Delta S>0)$, as the monomers lose degrees of freedom in the assembled as compared to the disassembled state.

Figure 2d,e (solid black, blue and red lines) shows the $\Delta G_{\text {stack }}, \Delta H^{*}{ }_{\text {stack }}$ and $-T \Delta S_{\text {stack }}$ collected values for the different size prestacked BTA assemblies. The energy data demonstrate self-assembly cooperativity. In particular, the perBTA self-assembly free-energy $\Delta G_{\text {stack }}-13 \mathrm{kcal} \mathrm{mol}^{-1}$ for the smaller $\mathbf{2} \mathbf{B T} \mathbf{A}_{\text {stack }}$ system, is seen to become rapidly more favorable for increasing stack sizes. Starting from the $1_{14 B A_{\text {stack }}}$ system, $\Delta G_{\text {stack }}$ tends to converge to a value perBTA of $\sim-30 \mathrm{kcal} \mathrm{mol}^{-1}$ (Figure 2e). This behavior is consistent with previous studies on cooperativity of the BTA assembly in vacuum and in organic solvent. ${ }^{22 b, 24-27}$ Cooperativity is also reflected by the $\Delta G_{\text {stack }}$ components reported in Figure $2 \mathrm{~d}$, where favorable $\Delta H^{*}$ stack and unfavorable $-T \Delta S_{\text {stack }}$ terms, respectively, converge to $\sim-50$ and $\sim+20 \mathrm{kcal} \mathrm{mol}^{-1}$ for sizes $\geq \mathbf{1 4 B T A}_{\text {stack. }}$. Interestingly, $\Delta G_{\text {stack, }}, \Delta H_{\text {stack, }}^{*}$ and $-T \Delta S_{\text {stack }}$ values for $\mathbf{1 4 B T A}_{\text {stack }}$ and $21 \mathbf{B T A}_{\text {stack }}$ are nearly identical to those of $48 \mathrm{BTA}_{\text {stack }}$ modeling an infinite BTA fiber. This means that above 14 BTA monomers, the system approaches the "polymer bulk conditions".

The $\Delta H^{*}$ term contains the BTA-BTA interaction energies (van der Waals and electrostatic interactions, also including $\mathrm{H}$ - 
bonding), but also the interaction of the BTAs with the solvent (hydrophobic effects). While we learn from the $\Delta H^{*}$ stack plot in Figure $2 \mathrm{~d}$ that globally these interactions become more favorable growing larger stacks, at least up to $\mathbf{1 4 B T A}_{\text {stack }}$ little can be said at this stage on the modulation of the fundamental interactions in the assemblies and on the role that these play on the growth of an ordered supramolecular BTA polymer in solution.

Ordered versus Disordered BTA Assemblies. MD simulation of an individual BTA monomer in water solution shows that the long side chains fold around the BTA core to minimize the hydrophobic surface exposed to the solvent (Figure 1d). ${ }^{20}$ To compare to the ordered BTA polymers, we systematically built additional BTA model systems in which multiple copies of individual and disassembled BTA monomers were randomly placed in a simulation box filled with explicit water molecules. In this way, we created molecular systems containing $2,3,5,7,14,21$, and 48 pre-equilibrated BTA monomers initially dispersed in solution (2BTA $_{\text {rand }}$ to 48BTA $\left._{\text {rand }}\right)$. These systems also underwent $400 \mathrm{~ns}$ of $\mathrm{MD}$ simulation in NPT conditions. During this simulation time, the BTA monomers aggregated in solution forming disordered BTA aggregates (Figures 1g, 2c, and S6b) that remained stable during the MD runs (see the Supporting Information).

Notably, the disordered BTA aggregates produced by these $\mathrm{MD}$ runs are local minimum energy conformations (in the real system, these should reconfigure into ordered stacks) in which the systems are trapped due to the intrinsic sampling limitations of the MD technique. Nevertheless, these disordered aggregates offer an interesting "far-from-equilibrium" comparison with the ordered $\mathbf{B T A} \mathbf{A}_{\text {stack }}$ systems. In fact, while in BTA $_{\text {rand }}$ systems the BTA monomers selfassemble mainly due to hydrophobic effects and nondirectional nonbond interactions (e.g., van der Waals), no persistent $\mathrm{H}$ bonding between the monomers is formed in these disordered aggregates. On the other hand, in $\mathbf{B T A}_{\text {stack }}$ systems, H-bonding intrinsically plays a key role.

Analysis of the self-assembly energies extracted from the MD simulations of these systems provided us with the $\Delta G_{\text {rand }}$, $\Delta H_{\text {rand }}^{*}$ and $-T \Delta S_{\text {rand }}$ data reported in Figure 2d,e (dotted black, blue and red lines). Traces of cooperative self-assembly are seen also in the case of disordered aggregates. This is reasonable, as some cooperativity can be expected due to the hydrophobic effects and the associated reduction in solventaccessible surface areas. $^{21 b, 33}$ The self-assembly energy for the random aggregates $\left(\Delta G_{\text {rand }}\right)$, converging to $\sim-22 \mathrm{kcal} \mathrm{mol}^{-1}$ per-BTA (Figure 2e: dotted black line), is found less favorable than for the ordered assemblies $\left(\sim-30 \mathrm{kcal} \mathrm{mol}^{-1}\right)$. This is largely due to a reduced $\Delta H^{*}$ rand term $\left(\sim-40 \mathrm{kcal} \mathrm{mol}^{-1}\right.$ for aggregates larger than 14 BTAs) as compared to that of BTA $_{\text {stack }}$ systems $\left(\sim-50 \mathrm{kcal} \mathrm{mol}^{-1}\right)$. Interestingly, the selfassembly solute entropic term $(-T \Delta S)$ is found invariant between ordered and disordered assemblies (Figure 2d: superimposed solid and dotted red lines), meaning that the BTA monomers lose the same number of degrees of freedom while self-assembling in an ordered rather than disordered way. Here, we find an interesting analogy with the component of self-assembly directly imputable to hydrophobic effects, generally thought of as to be due to entropy variations. ${ }^{34}$ In fact, hydrophobic effects are reasonably the same upon formation of ordered or disordered BTA assemblies (see below for additional evidence), consistent with a nearly identical self-assembly entropic term (see also methods section and Supporting Information).

Comparison of the $\Delta G_{\text {stack }}$ and $\Delta G_{\text {rand }}$ data provides another important result. The difference between the self-assembly freeenergies, $\Delta \Delta G=\Delta G_{\text {stack }}-\Delta G_{\text {rand }}$ indicates whether and to what extent formation of ordered BTA assemblies is energetically favored over that of disordered ones. Seen in Figure $2 f$, $\Delta \Delta G$ is found $>0$ for smaller aggregates (number of BTA $\leq 3$ : black circles), while it drops to $\Delta \Delta G \approx-6.7$ to $-10 \mathrm{kcal} \mathrm{mol}^{-1}$ for assembly sizes $\geq 5$ BTAs (red circles). This interesting evidence suggests that below a certain critical size, $\sim 5$ BTA based on our setup, formation of disordered BTA assemblies is favored over that of small ordered BTA stacks. On the other hand, above this threshold ( $\geq 5 \mathrm{BTA}$ ), there is a rather constant free-energy gain, on average $\Delta \Delta G=-8.4 \mathrm{kcal} \mathrm{mol}^{-1}$ per-BTA monomer, favoring formation of stacked assemblies over disordered ones. Importantly, this effect is found to be largely ascribed to $\Delta H^{*}$ (Figure $2 \mathrm{~d}$ ), and thus to enthalpic effects (see methods section). Figure $2 \mathrm{~g}$ reports a scheme illustrating the growth mechanism of ordered BTA supramolecular polymers in water that can be deduced purely on the basis of these thermodynamic observations, and regardless of the kinetic effects that, although important in the self-assembly mechanism, cannot be ascertained from such atomistic-resolution simulations: self-assembly of (i) initially dispersed BTA monomers induces (ii) formation of small disordered BTA aggregates that, when (iii) reaching the critical size ( $~ 5$ BTA), evolve toward ordered oligomers (iv). Further self-assembly of these ordered oligomers results in growth of a supramolecular polymer (v).

This mechanism finds consistency with experimental observations. In fact, upon injection of the BTA monomers from methanol (molecularly dissolved) into water, an initial UV spectrum is observed, which changes in time into the final spectrum obtained for the BTA polymers. ${ }^{17}$ Also, recent temperature-dependent studies on sugar-decorated BTA variants showed that at high temperature small aggregates are formed, which upon cooling are then converted into supramolecular polymers. ${ }^{23}$ These examples support the general mechanism for polymer growth shown in Figure $2 \mathrm{~g}$.

Key Interactions in BTA Self-Assembly. Seeing that the emergence and amplification of order in the BTA assemblies are controlled by enthalpic effects, that is, the formation of ordered BTA stacks is favored over that of disordered aggregates by increasingly favorable $\Delta H^{*}$ (Figure $2 \mathrm{~d}$ ), and considering that $\Delta H^{*}$ encompasses both solute-solute and solute-solvent interactions, immediate questions arising at this point are: Is order amplification in the polymer mainly controlled by hydrophobic effects or by BTA-BTA interactions? Is this mostly due to van der Waals or electrostatic interactions? What is the exact role of H-bonding in the growth of an ordered polymer?

The first step was to identify indicators that could be unambiguously ascribed to hydrophobic effects or to the different types of BTA-BTA interactions. A useful indicator of the strength of the hydrophobic effect is the solvent-accessible surface area (SASA) of the BTAs, and in particular the variation of the BTA SASA in the assembled or disassembled states. In fact, while aggregating in water, the BTA monomers reduce the amount of surface exposed to the solvent (Figure 3a: SASA shrinkage). The SASA data reported in Figure $3 \mathrm{~b}, \mathrm{c}$ are extracted from the MD simulations of the smaller (Figure $3 \mathrm{~b}$ : 
a

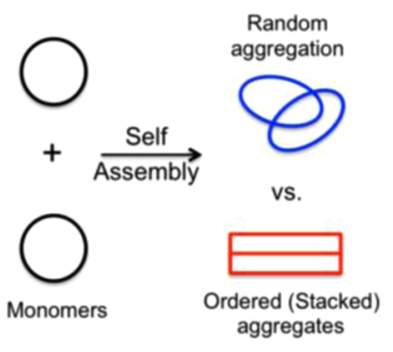

e

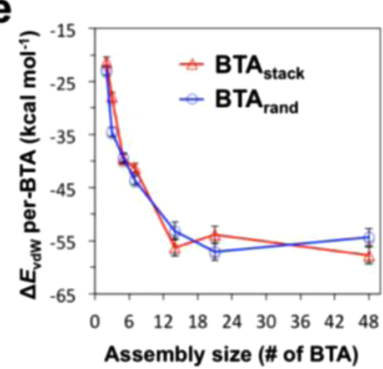

b

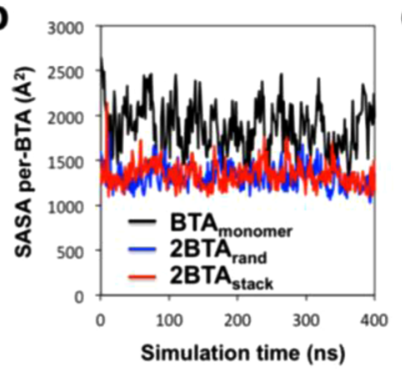

f

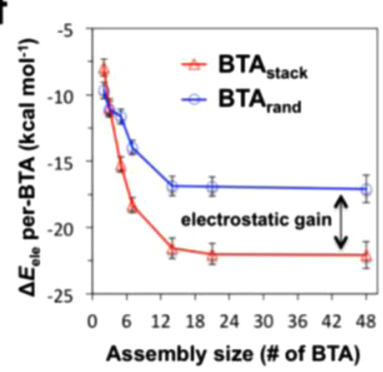

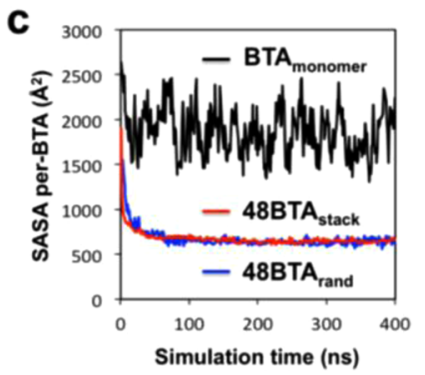

d

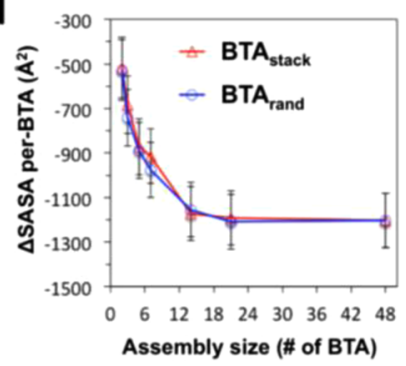

h

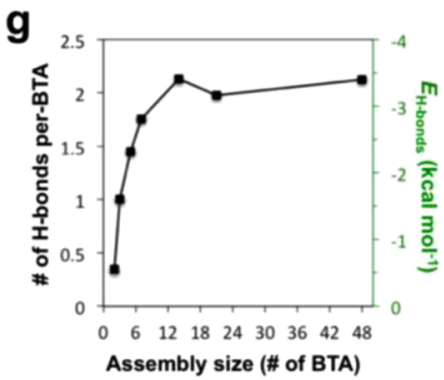

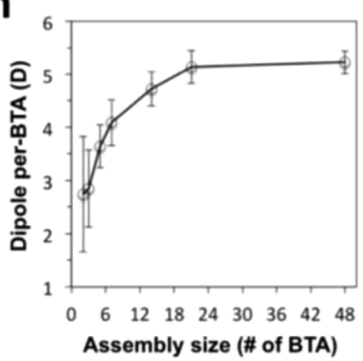

Figure 3. Dissecting fundamental interactions in BTA self-assembly. (a) Conceptual scheme used to interpret hydrophobic aggregation. Dispersed BTA monomers aggregate hydrophobically to decrease the surface (black circles) exposed to the surface. (b,c) Two examples: average solventaccessible surface area (SASA) of the BTAs in (a) ordered $\mathbf{2} \mathbf{B T A}_{\text {stack }}$ (red) and disordered $\mathbf{2 B T A}$ rand (blue), or (c) ordered 48BTA $\mathbf{A t a c k}_{\text {sed) and }}$

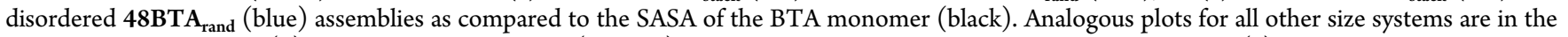
Supporting Information. (d) SASA variation per BTA ( $\triangle S A S A)$ for all cases as a function of the size of the assembly. (e) van der Waals interactions $\left(\Delta E_{\mathrm{vdW}}\right)$ between the BTAs in the assemblies. As with $\Delta$ SASA (d), also $\Delta E_{\mathrm{vdW}}$ data show characteristic cooperativity while growing larger aggregates, and negligible difference between ordered $\left(\mathbf{B T A}_{\text {stack }}\right.$ : red) and disordered (BTA rand $:$ blue) aggregates. (f) Electrostatic interactions $\left(\Delta E_{\text {ele }}\right)$ between the BTAs in the assemblies, showing clear differences between the two systems. $(\mathrm{g}$ ) Average number of H-bonding per-BTA in the BTA stack assemblies (black axis) and related H-bonding energy estimated by the average energy per-single $\mathrm{H}$-bond in aqueous solution for peptidic structures $\left(\sim-1.58 \mathrm{kcal} \mathrm{mol}^{-1}\right) \cdot^{20,36}(\mathrm{~h})$ Amplification of the dipole moment of the BTAs as a function of the size of the assembly calculated for BTA $_{\text {stack }}$ systems.

$\mathbf{2 B T A}_{\text {stack }}$ in red and $\mathbf{2} \mathbf{B T A} \mathbf{A}_{\text {rand }}$ in blue) and the larger BTA systems (Figure 3c: 48BTA stack $_{\text {in }}$ red and $48 \mathrm{BTA}_{\text {rand }}$ in blue).

It is interesting to compare these data with the SASA of the disassembled BTA monomer (in black). It is evident that the BTAs reduce their SASA upon aggregation, and this effect is much stronger in the larger assemblies than in the smaller ones (SASA data for all simulated systems are provided in the Supporting Information). Shown in Figure 3d, the per-BTA SASA shrinkage accompanying self-assembly (calculated as $\Delta S A S A=$ SASA $_{\text {assembly }}-$ SASA $\left._{\text {monomer }}\right)$ is plotted as a function of the aggregate size for all simulated systems. $\triangle$ SASA becomes more negative while increasing the size of the aggregate and converges for aggregate sizes $\geq 1$ 14BTA, demonstrating the cooperativity expected for hydrophobic aggregation mentioned above. Interestingly, this analysis demonstrates that the $\triangle$ SASA data (SASA shrinkage) are nearly identical in the ordered and disordered assemblies, which is also consistent with our conclusions on hydrophobic aggregation and entropy variations. These results suggest that hydrophobic and entropic effects taken alone are not responsible for preferential formation of ordered directional BTA aggregates versus disordered random ones in solution, thus indicating that the origin for the growth of ordered supramolecular polymers in water must be searched elsewhere.

We then focused on the nonbond terms of the global solutesolute BTA interaction $\left(\Delta E_{\text {gas }}\right)$. Shown in Figure 3e, the van der Waals interactions $\left(\Delta E_{\mathrm{vdW}}\right)$, while cooperative, do not discriminate between ordered and disordered assemblies, similar to entropic and hydrophobic terms. On the other hand, BTA-BTA electrostatic interactions $\left(\Delta E_{\text {ele }}\right)$ are augmented in the ordered (stacked) assemblies as compared to disordered ones, by $\sim 30 \%$ for sizes $\geq$ 14BTA (Figure $3 \mathrm{f}$ ). This electrostatic gain $\left(\Delta \Delta E_{\text {ele }}\right)$ of $\sim-5 \mathrm{kcal} \mathrm{mol}^{-1}$ per-BTA captures most of the global $\Delta \Delta G$ of Figure $2 \mathrm{f}$, revealing the main energetic factor responsible for the growth of ordered BTA stacks in solution.

While $\Delta \Delta E_{\text {ele }}$ identifies the electrostatic gain to ordered BTA assemblies, clearly accounting for the presence of the 3-fold $\mathrm{H}$ bonding between the BTA cores, this also includes other factors such as a more favorable electrostatic environment generated by the initial ordered displacement of the BTA cores, amplification of dipole-dipole interactions, and formation of macrodipoles. $^{25,27,35}$

We calculated from the MD simulation of the stacked assemblies the average number of $\mathrm{H}$-bonds per-BTA in the stacks and the associated H-bonding energy contribution $\left(E_{\mathrm{H}-\mathrm{bonds}}\right)$. As the atomistic force field used for this study does not contain an explicit term for $\mathrm{H}$-bonding, $E_{\mathrm{H} \text {-bonds }}$ was estimated by multiplying the average number of $\mathrm{H}$-bonds in each system for the energy per-single $\mathrm{H}$-bond in aqueous solution for peptidic structures $\left(\sim-1.58 \mathrm{kcal} \mathrm{mol}^{-1}\right) .^{20,36}$ Figure $3 \mathrm{~g}$ shows that the average number of H-bonding perBTA rapidly increases from $\sim 0.3-1$ in the case of the smaller and unstable stacks $\left(\mathbf{2 B T A}_{\text {stack }}\right.$ and $\left.3 \mathbf{B T A}_{\text {stack }}\right)$ to $\sim 2.2$ for stacks sizes $\geq \mathbf{1 4 B T A}_{\text {stack. }}$. Consistently, in the latter cases, the approximated $\mathrm{H}$-bonding energy contribution was found to reach a maximum of $\sim-3.4 \mathrm{kcal} \mathrm{mol}^{-1}$ per-BTA, equal to $\sim 70 \%$ of the global electrostatic gain $\Delta \Delta E_{\text {ele }}$. As discussed in detail in our recent work, ${ }^{20}$ the number of H-bonds per-BTA does not reach the maximum value of 3 in these systems due to 

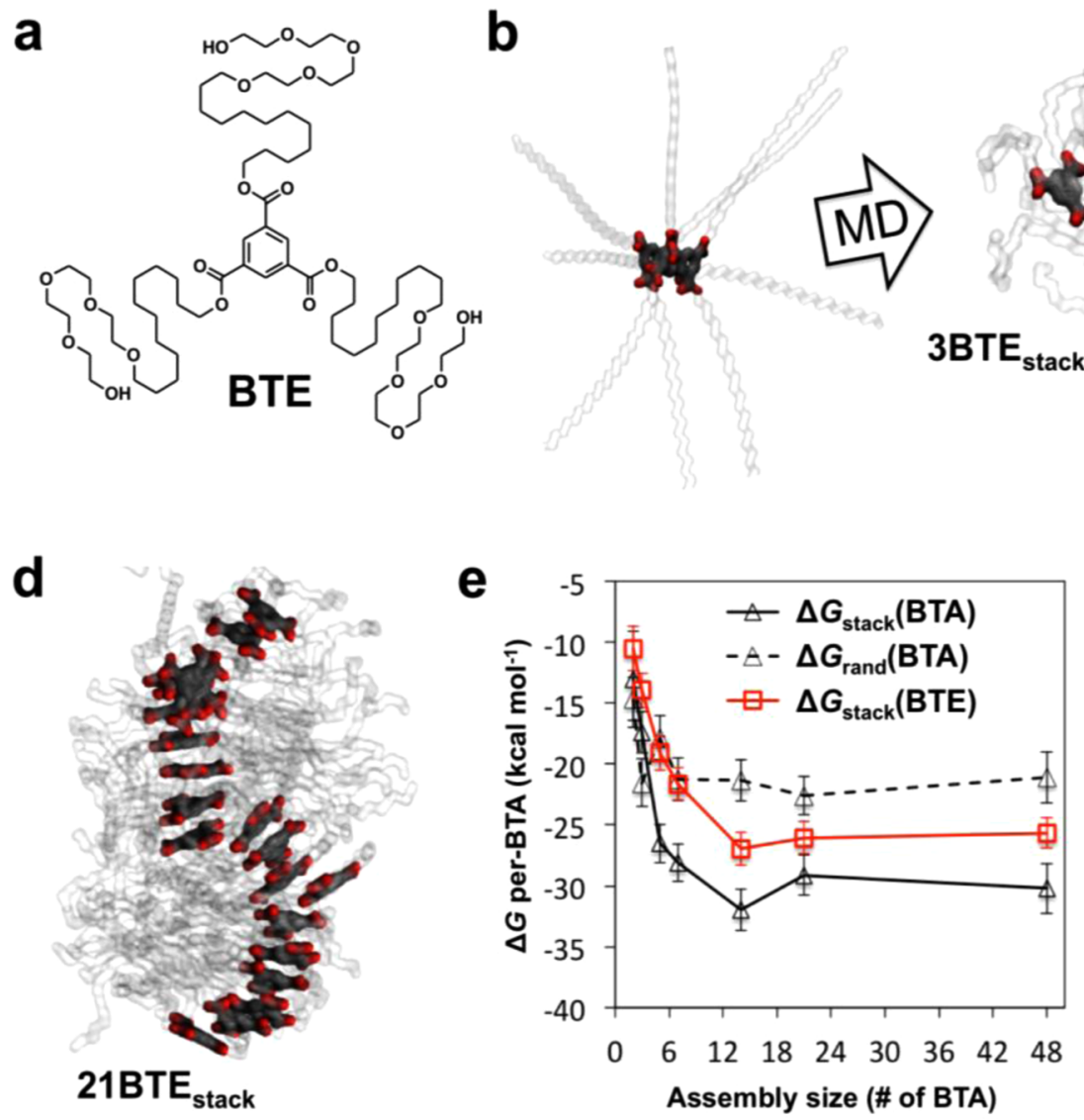

Figure 4. Modeling BTE stacks. (a) Molecular structure of the BTE monomer. (b) Starting (extended) and equilibrated structures taken form the $\mathrm{MD}$ simulation of $3 \mathrm{BTE}_{\text {stack }}$ system. (c,d) Equilibrated structures obtained form the MD simulations of $7 \mathbf{B T E}_{\text {stack }}(\mathrm{c})$ and $21 \mathrm{BTE}$ stack $(\mathrm{d})$ models.

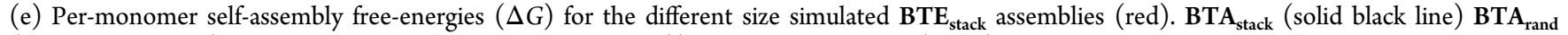
(dotted black lines) $\Delta G$ data are provided for comparison. (f) Per-BTA difference $(\Delta \Delta G)$ between the self-assembly free-energies of BTA $_{\text {stack }}$ $\left(\Delta G_{\text {stack }}(\mathrm{BTA})\right)$ and $\mathbf{B T E}_{\text {stack }}\left(\Delta G_{\text {stack }}(\mathrm{BTE})\right)$ assemblies of various sizes. Negative $\Delta \Delta G$ values indicate the favorable contribution to the stacked assemblies brought by $\mathrm{H}$-bonding.

the interaction with water, where the latter can (i) directly interfere with BTA-BTA H-bonding and (ii) generate local breakages/defects in the stacking following to the structural rearrangements of the assemblies in the aqueous medium (hydrophobic effects). ${ }^{20}$

From the $\mathrm{MD}$ simulations, we also calculated the average dipole moment of the BTAs in the stacks. Consistent with the $\mathrm{H}$-bonding data and with previous simulations of smaller BTA derivatives in an apolar solvent, ${ }^{25,27}$ the BTA dipole moment is seen to be amplified while growing with the stack size until a plateau is reached from $\sim \mathbf{2 1 B T A}$ stack (Figure $3 \mathrm{~h}$ ). While these analyses clearly indicate the amplification of electrostatic interactions along the stacks as the main factor leading to the growth of ordered BTA supramolecular polymers in water, we performed a last step to unambiguously isolate the effect of $\mathrm{H}$ bonding in the process.

Effect of $\mathrm{H}$-Bonding. We synthesized and investigated a 1,3,5-benzenetriester (BTE) derivative identical to BTA but having the amides replaced by ester groups (Figure $4 a$, see the Supporting Information for full synthetic information). As compared to the BTA monomers studied herein, BTE monomers lack the ability to form a H-bonding network between the cores in the supramolecular polymer while preserving the same hydrophobicity and structure of the cores. We compared the assemblies formed by these monomer variants in solution by means of $\mathrm{MD}$ simulations and experiments.

We built molecular models for BTE stacks $\left(\right.$ BTE $\left._{\text {stack }}\right)$ of sizes analogous to those created and simulated for the BTA case $\left(\mathbf{B T A}_{\text {stack }}\right)$. In this way, we obtained seven initially extended models for BTE stacks from $2 \mathrm{BTE}_{\text {stack }}$ to $48 \mathrm{BTE}_{\text {stack }}$. Analogous to $48 \mathrm{BTA}_{\text {stack, }}$ the larger $48 \mathrm{BTE}_{\text {stack }}$ system was also built to model an infinite BTE polymer through periodic replication of the 48 initially extended BTE monomers along the main axis of the fiber. The force field parameters for the BTE monomers were obtained according to the same procedure previously used for the BTA variants ${ }^{20}$ and other similar three-branched stacking discotic units (see methods section and Supporting Information for details). ${ }^{37}$ Each BTE stack model was simulated for $400 \mathrm{~ns}$ of NPT MD in explicit water molecules as done for the $\mathbf{B T A}_{\text {stack }}$ systems.

Initially, we guessed that the lack of $\mathrm{H}$-bonding would have made BTE monomers produce only random nondirectional aggregates in water, and that this would have been reflected by strong instability in the BTE stacks during the MD simulations. However, while complete stacking destabilization was seen for

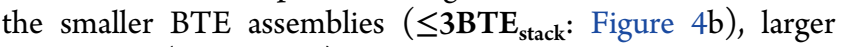
BTE stacks $\left(\geq\right.$ BBTE $\left._{\text {stack }}\right)$ were found to possess a certain level of intrinsic stability during the $\mathrm{MD}$ simulations, albeit to a lower extent compared to $\mathbf{B T A}_{\text {stack }}$ systems. For example, it is interesting to note in Figure 4c,d that while core-core stacking 

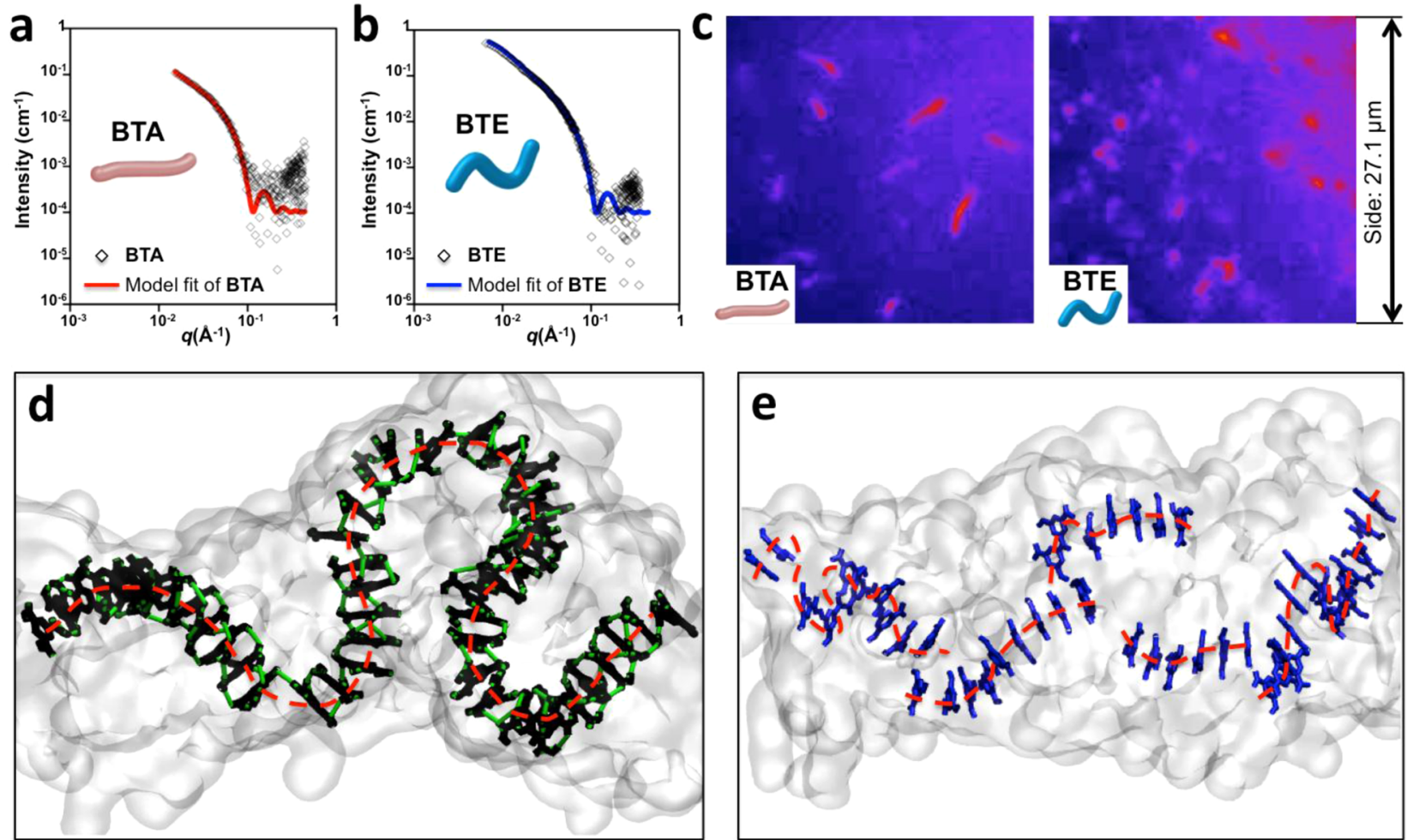

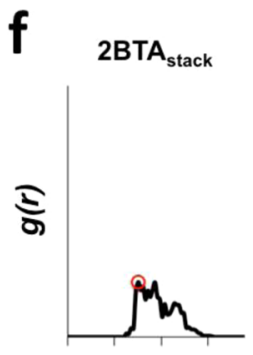

c 2c $3 c$

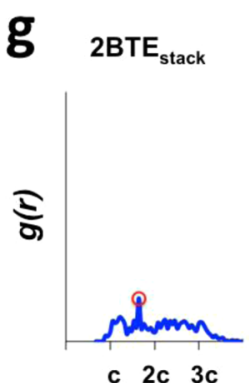

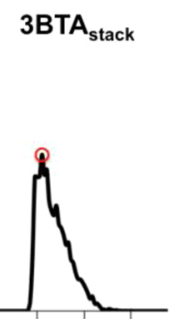

c 2 c $3 c$

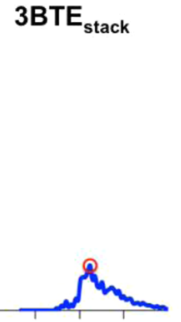

c $2 c 3 c$

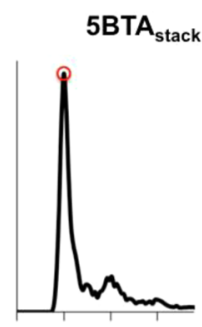

c 2c 3c

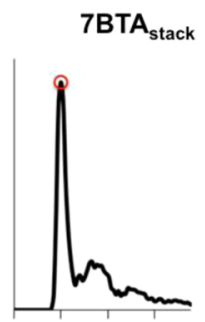

c 2c 3c

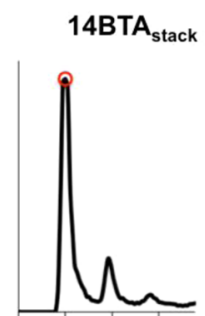

c 2c 3c

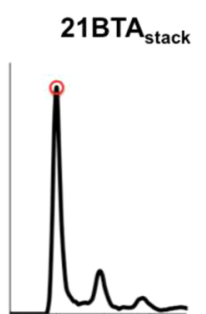

c 2c 3c

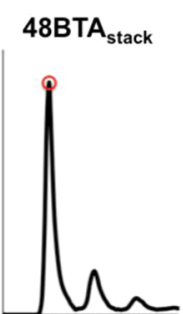

c 2c 3c

Figure 5. BTE versus BTA supramolecular polymers. (a,b) Experimental SAXS profiles of (a) BTA $\left(0.45\right.$ wt $\%$ in $\left.\mathrm{H}_{2} \mathrm{O}\right)$ and (b) BTE (0.45 wt $\%$ in $\mathrm{H}_{2} \mathrm{O}$ ) assemblies, fit with the Schurtenberger-Pedersen form factor. (c) Fluorescence microscopy images of BTA and BTE assemblies from $10 \mu \mathrm{M}$ aqueous solutions diluted to $0.5 \mu \mathrm{M}$ for imaging. (d,e) Detail of BTA core stacking taken from the MD simulation of the 48BTA stack system. BTA cores are colored in black, H-bonding in green, and the fiber is represented as transparent surface. (e) Detail of BTA core stacking taken from the MD simulation of the $\mathbf{4 8 B T E}$ stack system. BTE cores are colored in blue. Red dotted lines are provided to guide the eye. (f,g) Radial distribution functions $g(r)$ of the BTA (f: black) and BTE (g: blue) cores along the fibers calculated from the equilibrated phase MD trajectories (the last 100 ns of each MD run). Intercore spacing $c$ equals $3.4 \AA$. The $g(r)$ peaks indicative of stacking between neighbor BTA cores, $g(c)$, are identified by red circles.

is present in $7 \mathrm{BTE}_{\text {stack }}$ and $21 \mathrm{BTE}_{\text {stack }}$ systems, this seems to persist well only at short distance (stable stacks of $\sim 5$ BTEs), while defects appear in various spots along larger stacks. Because of the lack of the H-bonding network, stack bending during the MD simulations produces stacking instabilities and local slipping of the BTE cores with respect to each other. Also, all simulated $\mathrm{BTE}_{\text {stack }}$ assemblies showed negligible dipole moment (per-BTE value in the range $\sim 0-1 \mathrm{D}$ ). This can be rationally ascribed to the reduced intrinsic dipole of the ester groups compared to the amides of the BTA, while the absence of the H-bonding network in the BTE stacks makes the esters stay on the core ring planes.

As previously done for the BTA case, we calculated from the $\mathrm{MD}$ simulations the self-assembly free-energies for the $\mathbf{B T E}_{\text {stack }}$ 
systems. The plot of the $\Delta G_{\text {stack }}(\mathrm{BTE})$, in red in Figure $4 \mathrm{e}$, is shown to be nearly identical to that of the random BTA assemblies $\left(\mathbf{B T A}_{\text {rand }}\right)$ for sizes $\leq 7$ monomers. Above this limit $\left(\geq\right.$ 14BTE $\left._{\text {stack }}\right), \Delta G_{\text {stack }}(\mathrm{BTE})$ is found lying between the plots of $\Delta G_{\text {rand }}(\mathrm{BTA})$ and $\Delta G_{\text {stack }}(\mathrm{BTA})$. This is reasonable, as BTE $_{\text {stack }}$ stacks certainly lack the contribution of H-bonding as compared to $\mathbf{B T A}_{\text {stack }}$ assemblies, but as compared to $\mathbf{B T} \mathbf{A}_{\text {rand }}$ systems these benefit from a better arrangement of the cores (favorable electrostatic environment). This result indicates that formation of ordered assemblies is probable also in the case of BTE.

The difference $(\Delta \Delta G)$ between the self-assembly freeenergies of $\mathbf{B T A}_{\text {stack }}$ and $\mathbf{B T E} \mathbf{E}_{\text {stack }}$ systems is found to converge to $\sim-4 \mathrm{kcal} \mathrm{mol}^{-1}$ on average for stacks greater than 14 monomers (Figure 4f), well compatible with the contribution of H-bonding $\left(E_{\mathrm{H} \text {-bonds }} \approx-3.4 \mathrm{kcal} \mathrm{mol}^{-1}\right)$ found for the BTA stacks (vide supra, Figure $3 \mathrm{~g}$ ). Thus, the difference in freeenergy between $\mathbf{B T A}_{\text {stack }}$ and $\mathbf{B T E}_{\text {stack }}$ systems can be imputed in good approximation to the effect of $\mathrm{H}$-bonding. On the other hand, the difference in free-energy between BTE $_{\text {stack }}$ and BTA $_{\text {rand }}$ systems $\left(\Delta \Delta G\right.$ also $\left.\sim-4 \mathrm{kcal} \mathrm{mol}^{-1}\right)$, both lacking $\mathrm{H}$ bonding, can be ascribed to the beneficial effect of order into the core assembly (stacking), which makes the formation of BTE ordered assemblies favored over disordered ones also in the absence of H-bonding.

Experimentally, BTA and BTE self-assembly were compared in the milli- to micromolar regime using small-angle X-ray scattering (SAXS) and fluorescence microscopy experiments (Figure $5 \mathrm{a}-\mathrm{c}$ ). Both BTA and BTE variants form directional fibers in aqueous solution; yet the BTE fibers are found to be less rigid than the BTA.

The SAXS profiles obtained for BTA and BTE fibers (Figure $5 \mathrm{a}, \mathrm{b})$ are both found to fit well with a form factor describing semiflexible rods (Pedersen and Schurtenberger worm-like chain model). ${ }^{38}$ This model yields a cross-sectional radius of $\sim 3.3 \mathrm{~nm}$ for both fibers, while their persistence lengths differ considerably. The BTE fibers exhibit a relatively small Kuhn length $(\sim 8 \mathrm{~nm})$, while we obtain $\sim 27 \mathrm{~nm}$ for BTA fibers, indicating that the BTE fibers are more flexible than BTA fibers. This is further confirmed by the observation that BTA fibers can also be reliably fit by a rigid rod model, indicating a persistence length on the order of or beyond the experimental resolution $\left(\sim \pi / q_{\min }=45 \mathrm{~nm}\right.$, see the Supporting Information for details).

The differences in persistence length shown via SAXS are further supported by fluorescence microscopy. While previous fluorescence imaging of BTA fibers relied on (dynamic) covalent attachment of dyes, ${ }^{17,18}$ here we used Nile Red to label the hydrophobic interior of the assembled supramolecular systems in a noncovalent fashion. ${ }^{23}$ Movies in the Supporting Information show that BTE self-assembly produces less stiff, less ordered, and more polydisperse fibrous aggregates. Attempting to illustrate this via a snapshot (Figure 5c), one can see long, stiff, and defined fibers of the BTA system on the left in comparison to the poorly defined BTE system on the right. Surprisingly, during the BTE imaging, several long $(\mu \mathrm{m})$ and highly flexible fibers passed through the field of view; however, the majority of the sample was dominated by short and ill-defined aggregates.

The BTE self-assembly was also investigated utilizing UVvis spectroscopy, dynamic light scattering (DLS), and cryoTEM. The UV-vis showed little change upon self-assembly, the DLS experiments showed a monomodal distribution, while structures could not be visualized in cryo-TEM. These data and further discussion are reserved for the Supporting Information, but are attributed to the nonuniform self-assembly of the BTE derivative.

Taken altogether, these data reveal that while formation of directional assemblies and supramolecular polymers in water is possible also in the absence of $\mathrm{H}$-bonding, the latter has a noticeable effect on the persistence and rigidity of the supramolecular polymer.

Order Amplification. Additional analysis of the $\mathbf{B T A}_{\text {stack }}$ versus $\mathbf{B T E}_{\text {stack }}$ molecular models also supports the above conclusion. The radial distribution functions $(g(r))$ of the BTA and BTE cores extracted from the equilibrated phase MD simulations (the last $100 \mathrm{~ns}$ of each run) are useful indicators of the levels of order in monomer stacking. ${ }^{20,37}$ As the $g(r)$ measures the relative probability of finding neighbor BTA cores at stacking distance ( $c$, closest neighbor; $2 c$, second neighbor; etc.), and these data are averaged for all monomers in the stacks, the relative height of the $g(r)$ plots at distance $c, 2 c, 3 c$, etc. provides interesting insight on the evolution/amplification of stacking order in the assemblies with increasing aggregate size from a structural point of view. In general, the higher are the $g(r)$ peaks at stacking distances $c, 2 c, 3 c$, the more ordered, stable, and persistent is the core stacking in the assembly.

Shown in Figure $5 \mathrm{f}$, the $g(r)$ plots of the BTA cores present the characteristic three peaks $g(c), g(2 c)$, and $g(3 c)$ typical of ordered stacking for aggregate size $\geq 14$ BTA $_{\text {stack }}$. Looking at the smaller BTA aggregates, absence of the $g(c)$ and $g(2 c)$ peaks indicates that no ordered stacking is present in $\mathbf{2} \mathbf{B T} \mathbf{A}_{\text {stack }}$ and $\mathbf{3 B T A}_{\text {stack }}$, consistent with the fact that these small stacks are seen to disassemble during the MD simulations. Starting from

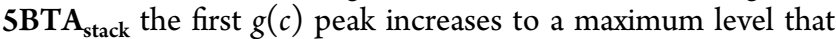
is nearly conserved also in the larger aggregates (red circles). It is interesting to note that the $g(r)$ plots of $\mathbf{5 B T A}_{\text {stack }}$ and $\mathbf{7 B T A}_{\text {stack }}$ possess clear first $g(c)$ peak, but no clear $g(2 c)$ and $g(3 c)$ peaks (stacking order present only up to the first closest neighbor), indicating that these two systems are still not persistent as full stacks. On the contrary, amplification and consistency of the $g(r)$ plots and of $g(c), g(2 c)$, and $g(3 c)$ peaks in $14 \mathrm{BTA}_{\text {stack }}$ 21BTA stack $_{\text {, }}$ and $48 \mathrm{BTA} \mathrm{A}_{\text {stack }}$ systems demonstrate that the stacking order along the fiber is approaching the maximum level for stack sizes $\geq$ 14BTA $_{\text {stack}}$.

Our free-energy data show that over a critical size the spontaneous growth of ordered BTA assemblies is favored over that of disordered aggregates. At the same time, the above $g(r)$ data demonstrate that overall stacking order into these assemblies increases with their size. These structural and energetic analyses provide a picture where, as an energetically favored event, stacking order emerges into the small BTA aggregates, increases, and tends to level off starting from oligomer size $\geq \mathbf{1 4 B T A}_{\text {stack}}$ : that is, order amplification.

Interestingly, the same analysis for the $\mathbf{B T E}_{\text {stack }}$ systems (Figure $5 \mathrm{~g}$ ) shows that while for assembly sizes $\leq \mathbf{5 B T E}$ stack $_{\text {the }}$ $g(r)$ plots are in good approximation consistent with those of BTA $_{\text {stack }}$ ones, starting from sizes $\geq 7 \mathbf{B T E}_{\text {stack }}$ the heights of the $g(r)$ plots drop dramatically. The $g(r)$ decrease seen for larger $\mathrm{BTE}_{\text {stack }}$ systems can be imputed to the numerous defects emerging in the BTE stacking over a certain stack length. This observation is fully consistent with the experimental persistence length ( $\sim 4 \mathrm{~nm}$ for the BTE), indicating that stacking persistence into BTE fibers is reduced as compared to BTA ones. This data would correspond to $\sim 11-12$ monomers (considering a stacking distance of $3.4 \AA$ ), while over this limit 
the BTE fibers are not persistent. As seen in Figure 4c,d, BTE stacks seem to preserve some short-range order imputable to the above-mentioned order effect, but fail in real order amplification when the stacks grow. Figure 5 d,e provides visual inspection inside the $48 \mathrm{BTA}_{\text {stack }}$ and $48 \mathrm{BTE}_{\text {stack }}$ simulated systems, showing that while core stacking is uniform inside the BTA polymer, the BTE core stacking breaks into shorter segments. This effect is consistent with the enhanced flexibility seen in the experiments for BTE assemblies as compared to BTA ones, and is directly imputable to the lack of $\mathrm{H}$-bonding in the BTE assembly.

\section{CONCLUSIONS}

Focusing on 1,3,5-benzenetricarboxamide (BTA), a selfassembling motif forming supramolecular polymers in water, we have designed an ad hoc molecular simulation strategy to study in detail the factors triggering and controlling the formation of an ordered assembly in aqueous environment, where an intricate interplay of hydrophobic and monomermonomer nonbond interactions takes place. Systematic comparison of ordered (stacked) and disordered BTA assemblies of the same size (number of monomers) indicates that the aggregates need to reach a critical size to favor the formation of ordered stacks over disordered aggregates. Detailed decomposition of the self-assembly energies demonstrates that the amplification of order in the assemblies and consequent growth of an ordered supramolecular polymers in water are mainly due to electrostatic effects. To isolate the effect of H-bonding, we compared the BTA assemblies with those formed by a 1,3,5-benzenetricarboxyester (BTE) variant by means of experiments and MD simulations. BTE monomers differ only by having the amide groups replaced by esters, thus lacking the ability to form the $\mathrm{H}$-bonding network between the monomers. Nevertheless, these were also found to form fibrous assemblies in water, but BTE fibers were found more flexible and less persistent than BTA supramolecular polymers. This work provides a unique picture of the role of $\mathrm{H}$-bonding in the self-assembly of supramolecular polymers in water. Our results unambiguously demonstrate that order amplification in the fibers and the growth of a persistent directional supramolecular polymer in water solution are greatly controlled by $\mathrm{H}$-bonding. This approach allows one to directly relate the structure of the monomer to the structure and properties of the supramolecular polymer that these form in water.

\section{ASSOCIATED CONTENT}

\section{S Supporting Information}

The Supporting Information is available free of charge on the ACS Publications website at DOI: 10.1021/jacs.6b07530.

Details of the simulated molecular systems, computational methods for parametrization, simulation, and structural and energetic analyses of the models; additional data and figures from the MD simulations; synthesis procedure for BTE monomers, and SAXS and fluorescent microscopy methods (PDF)

Time-lapse imaging (AVI)

Time-lapse imaging (AVI)

Time-lapse imaging (AVI)

Time-lapse imaging (AVI)

\section{AUTHOR INFORMATION}

\section{Corresponding Author}

*giovanni.pavan@supsi.ch

\section{Notes}

The authors declare no competing financial interest.

\section{ACKNOWLEDGMENTS}

G.M.P. acknowledges the support from the Swiss National Science Foundation (SNSF grant 200021 162827). M.B.B. acknowledges the Province of Limburg. I.K.V. acknowledges the Dutch Science Foundation (NWO ECHOSTIP Grant 717.013.005, NWO VIDI Grant 723.014.006). L.A. acknowledges the AXA research fund. The Dutch Ministry of Education, Culture and Science (Gravity program 024.001.035) is acknowledged for funding. We thank René Lafleur (ICMS) for his support in cryo-TEM of BTE.

\section{REFERENCES}

(1) Yang, L.; Tan, X.; Wang, Z.; Zhang, X. Chem. Rev. 2015, 115, $7196-7239$.

(2) Aida, T.; Meijer, E. W.; Stupp, S. I. Science 2012, 335, 813-817.

(3) Webber, M. J.; Appel, E. A.; Meijer, E. W.; Langer, R. Nat. Mater. 2015, 15, 13-26.

(4) Krieg, E.; Bastings, M. M. C.; Besenius, P.; Rybtchinski, B. Chem. Rev. 2016, 116, 2414-2477.

(5) Dankers, P. Y. W.; Harmsen, M. C.; Brouwer, L. A.; van Luyn, M. J. A.; Meijer, E. W. Nat. Mater. 2005, 4, 568-574.

(6) Lee, O.-S.; Stupp, S. I.; Schatz, G. C. J. Am. Chem. Soc. 2011, 133, $3677-3683$.

(7) Cui, H.; Webber, M. J.; Stupp, S. I. Biopolymers 2010, 94, 1-18.

(8) Matson, J. B.; Zha, R. H.; Stupp, S. I. Curr. Opin. Solid State Mater. Sci. 2011, 15, 225-235.

(9) (a) Muylaert, D. E. P.; Van Almen, G. C.; Talacua, H.; Fledderus, J. O.; Kluin, J.; Hendrikse, S. I. S.; Van Dongen, J. L. J.; Sijbesma, E.; Bosman, A. W.; Mes, T.; Thakkar, S. H.; Smits, A. I. P. M.; Bouten, C. V. C.; Dankers, P. Y. W.; Verhaar, M. C. Biomaterials 2016, 76, 187195. (b) Bastings, M. M. C.; Koudstaal, S.; Kieltyka, R. E.; Nakano, Y.; Pape, A. C. H.; Feyen, D. A. M.; Van Slochteren, F. J.; Doevendans, P. A.; Sluijter, J. P. G.; Meijer, E. W.; Chamuleau, S. A. J.; Dankers, P. Y. W. Adv. Healthcare Mater. 2014, 3, 70-78.

(10) Davis, M. E. Mol. Pharmaceutics 2009, 6, 659-668.

(11) Li, J.; Loh, X. J. Adv. Drug Delivery Rev. 2008, 60, 1000-1017.

(12) Appel, E. A.; Loh, X. J.; Jones, S. T.; Biedermann, F.; Dreiss, C. A.; Scherman, O. A. J. Am. Chem. Soc. 2012, 134, 11767-11773.

(13) Neirynck, P.; Brinkmann, J.; An, Q.; van der Schaft, D. W. J.; Milroy, L.-G.; Jonkheijm, P.; Brunsveld, L. Chem. Commun. 2013, 49, $3679-3681$

(14) (a) Garzoni, M.; Cheval, N.; Fahmi, A.; Danani, A.; Pavan, G. M. J. Am. Chem. Soc. 2012, 134, 3349-3357. (b) Astachov, V.; Garzoni, M.; Danani, A.; Choy, K.-L.; Pavan, G. M.; Fahmi, A. New J. Chem. 2016, 40, 6325-6331.

(15) Wang, J.; Xia, H.; Zhang, Y.; Lu, H.; Kamat, R.; Dobrynin, A. V.; Cheng, J.; Lin, Y. J. Am. Chem. Soc. 2013, 135, 11417-11420.

(16) Cantekin, S.; de Greef, T. F. A.; Palmans, A. R. A. Chem. Soc. Rev. 2012, 41, 6125-6137.

(17) Leenders, C. M. A.; Albertazzi, L.; Mes, T.; Koenigs, M. M. E.; Palmans, A. R. A.; Meijer, E. W. Chem. Commun. 2013, 49, 19631965.

(18) (a) Albertazzi, L.; van der Zwaag, D.; Leenders, C. M. A.; Fitzner, R.; Van der Hofstad, R. W.; Meijer, E. W. Science 2014, 344, 491-495. (b) Baker, M. B.; Gosens, R. P. J.; Albertazzi, L.; Matsumoto, N. M.; Palmans, A. R. A.; Meijer, E. W. ChemBioChem 2016, 17, 207-213.

(19) Albertazzi, L.; Martinez-Veracoechea, F. J.; Leenders, C. M. A.; Voets, I. K.; Frenkel, D.; Meijer, E. W. Proc. Natl. Acad. Sci. U. S. A. 2013, 110, 12203-12208. 
(20) Baker, M. B.; Albertazzi, L.; Voets, I. K.; Leenders, C. M. A.; Palmans, A. R. A.; Pavan, G. M.; Meijer, E. W. Nat. Commun. 2015, 6, 6234.

(21) (a) Leenders, C. M. A.; Baker, M. B.; Pijpers, I. A. B.; Lafleur, R. P. M.; Albertazzi, L.; Palmans, A. R. A.; Meijer, E. W. Soft Matter 2016, 12, 2887-2893. (b) Fu, I. W.; Markegard, C. B.; Chu, B. K. C.; Nguyen, H. D. Langmuir 2014, 30, 7745-7754. (c) Paramonov, S. E.; Jun, H.-W.; Hartgerink, J. D. J. Am. Chem. Soc. 2006, 128, 7291-7298.

(22) (a) De Greef, T. F. A.; Smulders, M. M. J.; Wolffs, M.; Schenning, A. P. H.; Sijbesma, J. R.; Meijer, E. W. Chem. Rev. 2009, 109, 5687-5754. (b) Kulkarni, C.; Balasubramanian, B.; George, S. J. ChemPhysChem 2013, 14, 661-673.

(23) Leenders, C. M. A.; Jansen, G.; Frissen, M. M. M.; Lafleur, R. F. M.; Voets, I. K.; Palmans, A. R. A.; Meijer, E. W. Chem. - Eur. J. 2016, 22, 4608-4615.

(24) Filot, I. A. W.; Palmans, A. R. A.; Hilbers, P. A. J.; van Santen, R. A.; Pidko, E. A.; de Greef, T. F. A. J. Phys. Chem. B 2010, 114, 1366713674.

(25) Bejagam, K. K.; Fiorin, G.; Klein, M. L.; Balasubramanian, S. J. Phys. Chem. B 2014, 118, 5218-5228.

(26) Kulkarni, C.; Reddy, S. K.; George, S. J.; Balasubramanian, S. Chem. Phys. Lett. 2011, 515, 226-230.

(27) Albuquerque, R. Q.; Timme, A.; Kress, R.; Senker, J.; Schmidt, H.-W. Chem. - Eur. J. 2013, 19, 1647-1657.

(28) Chami, F.; Wilson, M. R. J. Am. Chem. Soc. 2010, 132, 77947802.

(29) Bejagam, K. K.; Balasubramanian, S. J. Phys. Chem. B 2015, 119, $5738-5746$.

(30) Smulders, M. M. J.; Schenning, A. P. H. J.; Meijer, E. W. J. Am. Chem. Soc. 2008, 130, 606-611.

(31) Jorgensen, W. L.; Chandrasekhar, J.; Madura, J. D.; Impey, R. W.; Klein, M. L. J. Chem. Phys. 1983, 79, 926-935.

(32) Case, D. A.; Darden, T. A.; Cheatham, T. E., III; Simmerling, C. L.; Wang, J.; Duke, R. E.; Luo, R.; Walker, R. C.; Zhang, W.; Merz, K. M.; Roberts, B..; Hayik, S.; Roitberg, A.; Seabra, G.; Swails, J.; Goetz, A. W.; Kolossvary, I.; Wong, K. F.; Paesani, F.; Vanicek, J.; Wolf, R. M.; Liu, J.; Wu, X.; Brozell, S.; Steinbrecher, T.; Gohlke, H.; Cai, Q.; Ye, X.; Wang, J.; Hsieh, M.-J.; Cui, G.; Roe, D. R.; Mathews, D. H.; Seetin, M. G.; Salomon-Ferrer, R.; Sangui, C.; Babin, V.; Luchko, T.; Gusarov, S.; Kovalenko, A.; Kollman, P. A. AMBER 12; University of California: San Francisco, CA, 2012.

(33) Hills, R. D., Jr.; Brooks, C. L., III J. Mol. Biol. 2007, 368, 894901.

(34) (a) Tanford, C. The Hydrophobic Effect; John Wiley and Sons: New York, 1980. (b) Chandler, D. Nature 2005, 437, 640-647.

(35) Kulkarni, C.; Bejagam, K. K.; Senanayak, S. P.; Narayan, K. S.; Balasubramanian, S.; George, S. J. J. Am. Chem. Soc. 2015, 137, 39243932.

(36) Sheu, S.-Y.; Yang, D.-Y.; Selzle, H. L.; Schlag, E. W. Proc. Natl. Acad. Sci. U. S. A. 2003, 100, 12683-12687.

(37) Beltran, E.; Garzoni, M.; Feringan, B.; Vancheri, A.; Barbera, J.; Serrano, J. L.; Pavan, G. M.; Gimenez, R.; Sierra, T. Chem. Commun. 2015, 51, 1811-1814.

(38) Pedersen, J. S.; Schurtenberger, P. Macromolecules 1996, 29, $7602-7612$. 